FACTA UNIVERSITATIS (NIŠ)

Ser. Math. Inform. Vol. 36, No 2 (2021), 449-459

https://doi.org/10.22190/FUMI210210033N

Original Scientific Paper

\title{
A NOTE ON SOME SYSTEMS OF GENERALIZED SYLVESTER EQUATIONS *
}

\section{Jovana Nikolov Radenković}

Faculty of Sciences and Mathematics, University of Niš, Serbia

\begin{abstract}
In this paper, we study two systems of generalized Sylvester operator equations. We derive necessary and sufficient conditions for the existence of a solution and provide the general form of a solution. We extend some recent resuts to more general settings.
\end{abstract}

Key words: Sylvester equations, generalized inverses, Matrix equations and identities

\section{Introduction}

Let $\mathcal{H}, \mathcal{K}, \mathcal{F}, \mathcal{G}, \mathcal{L}, \mathcal{M}, \mathcal{N}$ be complex Hilbert spaces and let $\mathcal{B}(\mathcal{H}, \mathcal{K})$ denote the set of all bounded linear operators from $\mathcal{H}$ to $\mathcal{K}$. For a given $A \in \mathcal{B}(\mathcal{H}, \mathcal{K})$, the symbols $\mathcal{N}(A)$ and $\mathcal{R}(A)$ denote the null space and the range of operator $A$, respectively. The identity operator is always denoted by $I$. If $A \in \mathcal{B}(\mathcal{H}, \mathcal{K})$ has a closed range, then there exists unique operator $X \in \mathcal{B}(\mathcal{K}, \mathcal{H})$ satisfying the following equations

$$
\text { (1) } A X A=A \text { (2) } X A X=X \quad(3)(A X)^{*}=A X \quad \text { (4) }(X A)^{*}=X A \text {. }
$$

Such operator is called the Moore-Penrose inverse of an operator $A \in \mathcal{B}(\mathcal{H}, \mathcal{K})$ which is denoted by $A^{\dagger}$. If $X \in \mathcal{B}(\mathcal{K}, \mathcal{H})$ satisfies the equation (1), i.e. $A X A=A$, then $X$ is an inner generalized inverse of $A$, and is usually denoted by $A^{-}$. For $A \in \mathcal{B}(\mathcal{H}, \mathcal{K})$ there exists a Moore-Penrose inverse, if and only if there exists its

Received February 10, 2021; accepted May 03, 2021

Communicated by Dragana Cvetković Ilić

Corresponding Author: Jovana Nikolov Radenković, Faculty of Sciences and Mathematics, University of Niš, Serbia | E-mail: jovana.nikolov@gmail.com

2010 Mathematics Subject Classification. Primary 15A09; Secondary 15A24

(C) 2021 by University of Niš, Serbia | Creative Commons LiCense: CC BY-NC-ND

*The author is supported by the Ministry of Education, Science and Technological Development, Republic of Serbia (451-03-9/2021-14/200124). 
inner generalized inverse if and only if $\mathcal{R}(A)$ is closed. In this case, we say that $A$ is regular. Furthermore, $L_{A}$ and $R_{A}$ stand for two projections $L_{A}=I-A^{\dagger} A$ and $R_{A}=I-A A^{\dagger}$. induced by $A$, respectively.

In this paper, we study two systems of generalized Sylvester operator equations

$$
A_{1} X_{1}-X_{2} B_{1}=C_{1}, \quad A_{2} X_{3}-X_{2} B_{2}=C_{2},
$$

where $A_{1} \in \mathcal{B}(\mathcal{H}, \mathcal{K}), B_{1} \in \mathcal{B}(\mathcal{F}, \mathcal{G}), C_{1} \in \mathcal{B}(\mathcal{F}, \mathcal{K}), A_{2} \in \mathcal{B}(\mathcal{M}, \mathcal{K}), B_{2} \in \mathcal{B}(\mathcal{L}, \mathcal{G})$, $C_{2} \in \mathcal{B}(\mathcal{L}, \mathcal{K})$, and

$$
A_{1} X_{1}-X_{2} B_{1}=C_{1}, \quad A_{2} X_{2}-X_{3} B_{2}=C_{2},
$$

where $A_{1} \in \mathcal{B}(\mathcal{H}, \mathcal{K}), B_{1} \in \mathcal{B}(\mathcal{F}, \mathcal{G}), C_{1} \in \mathcal{B}(\mathcal{F}, \mathcal{K}), A_{2} \in \mathcal{B}(\mathcal{K}, \mathcal{M}), B_{2} \in \mathcal{B}(\mathcal{G}, \mathcal{N})$, $C_{2} \in \mathcal{B}(\mathcal{G}, \mathcal{M})$.

Systems of such type of matrix equations have been considered by many authors $[3,4,5,6,7]$. In this pape,r we extended recent results [7] on systems of quaternion matrix equations to infinite dimensional settings and provide much simpler proofs to existing conditions.

\section{Main results}

The following two lemmas play a key role in this paper:

Lemma 2.1. [1] Let $A \in \mathcal{B}(\mathcal{H}, \mathcal{K}), B \in \mathcal{B}(\mathcal{F}, \mathcal{G})$ and $C \in \mathcal{B}(\mathcal{F}, \mathcal{K})$ be such that $\mathcal{R}(A)$ and $\mathcal{R}(B)$ are closed. Then the operator equation

$$
A X B=C
$$

is consistent if and only if

$$
A A^{-} C B^{-} B=C,
$$

for some $A^{-}$and $B^{-}$, in which case the general solution is given by

$$
X=A^{-} C B^{-}+Y-A^{-} A Y B B^{-},
$$

for arbitrary $Y \in \mathcal{B}(\mathcal{G}, \mathcal{H})$.

Lemma 2.2. [2] Let $E, F, G, D, N, M$ be Banach spaces. Let $A_{1} \in \mathcal{B}(F, E), A_{2} \in$ $\mathcal{B}(F, N), B_{1} \in \mathcal{B}(D, G), B_{2} \in \mathcal{B}(M, G)$ and

$$
T:=\left(I_{G}-B_{1} B_{1}^{-}\right) B_{2} \quad \text { and } \quad S:=A_{2}\left(I_{F}-A_{1}^{-} A_{1}\right)
$$

be all regular. Moreover, let $A_{1} A_{1}^{-} C_{1} B_{1}^{-} B_{1}=C_{1}$ and $A_{2} A_{2}^{-} C_{2} B_{2}^{-} B_{2}=C_{2}$. Then the equations

$$
A_{1} X B_{1}=C_{1} \quad \text { and } \quad A_{2} X B_{2}=C_{2}
$$

have a common solution if and only if

$$
\left(I_{N}-S S^{-}\right) C_{2}\left(I_{M}-T^{-} T\right)=\left(I_{N}-S S^{-}\right) A_{2} A_{1}^{-} C_{1} B_{1}^{-} B_{2}\left(I_{M}-T^{-} T\right) .
$$


In this case, the general common solution is given by

$$
\begin{aligned}
X= & \left(A_{1}^{-} C_{1}-\left(I_{F}-A_{1}^{-} A_{1}\right) S^{-}\left(A_{2} A_{1}^{-} C_{1}-W\right)\right) B_{1}^{-}\left(I_{G}-B_{2} T^{-}\left(I_{G}-B_{1} B_{1}^{-}\right)\right) \\
& +\left(\left(I_{F}-\left(I_{F}-A_{1}^{-} A_{1}\right) S^{-} A_{2}\right) A_{1}^{-} V+\left(I_{F}-A_{1}^{-} A_{1}\right) S^{-} C_{2}\right) T^{-}\left(I_{G}-B_{1} B_{1}^{-}\right) \\
& +Z-\left(A_{1}^{-} A_{1}+\left(I_{F}-A_{1}^{-} A_{1}\right) S^{-} S\right) Z\left(B_{1} B_{1}^{-}+T T^{-}\left(I_{G}-B_{1} B_{1}^{-}\right)\right),
\end{aligned}
$$

where

$$
\begin{aligned}
V= & C_{1} B_{1}^{-} B_{2}\left(I_{M}-T^{-} T\right)+A_{1} A_{2}^{-}\left(I_{N}-S S^{-}\right) C_{2} T^{-} T+A_{1} A_{1}^{-} Q T^{-} T \\
& -A_{1} A_{2}^{-}\left(I_{N}-S S^{-}\right) A_{2} A_{1}^{-} Q T^{-} T, \\
W= & \left(I_{N}-S S^{-}\right) A_{2} A_{1}^{-} C_{1}+S S^{-} C_{2}\left(I_{M}-T^{-} T\right) B_{2}^{-} B_{1}+S S^{-} P B_{1}^{-} B_{1} \\
& -S S^{-} P B_{1}^{-} B_{2}\left(I_{M}-T^{-} T\right) B_{2}^{-} B_{1},
\end{aligned}
$$

in which $P, Q, Z$ are arbitrary elements of $\mathcal{B}(D, N), \mathcal{B}(M, E)$ and $\mathcal{B}(G, F)$, respectively.

Note that in the preceding lemmas, in the solvability conditions and formulas for general solutions, arbitrary inner generalized inverses can be replaced by the Moore-Penrose inverse. For example, in Lemma 2.1, if

$$
A A^{-} C B^{-} B=C
$$

holds for some $A^{-}$and $B^{-}$, then

$$
A A^{\dagger} C B^{\dagger} B=A A^{\dagger}\left(A A^{-} C B^{-} B\right) B^{\dagger} B=A A^{-} C B^{-} B=C .
$$

Conversly, if

$$
A A^{\dagger} C B^{\dagger} B=C
$$

holds, then for arbitrary $A^{-}$and $B^{-}$it follows

$$
A A^{-} C B^{-} B=A A^{-}\left(A A^{\dagger} C B^{\dagger} B\right) B^{-} B=A A^{\dagger} C B^{\dagger} B=C .
$$

So, for $A^{-}$and $B^{-}$in the solvability conditions and formulas for general solutions, we can choose exactly $A^{\dagger}$ and $B^{\dagger}$, respectively.

Theorem 2.1. Let $A_{1} \in \mathcal{B}(\mathcal{H}, \mathcal{K}), B_{1} \in \mathcal{B}(\mathcal{F}, \mathcal{G}), C_{1} \in \mathcal{B}(\mathcal{F}, \mathcal{K}), A_{2} \in \mathcal{B}(\mathcal{M}, \mathcal{K})$, $B_{2} \in \mathcal{B}(\mathcal{L}, \mathcal{G}), C_{2} \in \mathcal{B}(\mathcal{L}, \mathcal{K})$ be such that $A_{1}, A_{2}, B_{1}, B_{2}, S$ and $T$ are all regular. Put

$$
\begin{aligned}
& T=\left(I-B_{1} B_{1}^{\dagger}\right) B_{2}, \quad S=\left(I-A_{2} A_{2}^{\dagger}\right) A_{1} A_{1}^{\dagger} \\
& C=\left(I-A_{2} A_{2}^{\dagger}\right)\left(C_{2}-\left(I-A_{1} A_{1}^{\dagger}\right) C_{1} B_{1}^{\dagger} B_{2}\right)\left(I-T^{\dagger} T\right) .
\end{aligned}
$$

The following statements are equivalent:

(i) The system (1.1) is consistent; 
(ii) $R_{A_{1}} C_{1} L_{B_{1}}=0, R_{A_{2}} C_{2} L_{B_{2}}=0, R_{S} C=0$;

(iii) $R_{A_{1}} C_{1} L_{B_{1}}=0, C\left(I-\left(B_{2} L_{T}\right)^{\dagger}\left(B_{2} L_{T}\right)\right)=0, R_{S} C=0$.

In this case, the general solution to the system (1.1) is given by

$$
\begin{aligned}
X_{1}= & A_{1}^{\dagger} S^{\dagger}\left(R_{A_{1}} C_{1}+W\right) B_{1}^{\dagger} B_{1}+A_{1}^{\dagger} Z B_{1}-A_{1}^{\dagger} S^{\dagger} S Z B_{1}+A_{1}^{\dagger} C_{1}+L_{A_{1}} R, \\
X_{2}= & \left(-R_{A_{1}} C_{1}+S^{\dagger}\left(R_{A_{1}} C_{1}+W\right)\right) B_{1}^{\dagger}\left(I-B_{2} T^{\dagger}\right) \\
& +\left(\left(I-S^{\dagger}\right) R_{A_{1}} V-S^{\dagger} C_{2}\right) T^{\dagger}+Z-\left(I-A_{1} A_{1}^{\dagger}+S^{\dagger} S\right) Z\left(B_{1} B_{1}^{\dagger}+T T^{\dagger}\right), \\
X_{3}= & A_{2}^{\dagger}\left(-R_{A_{1}} C_{1}-S^{\dagger}\left(R_{A_{1}} C_{1}+W\right)\right) B_{1}^{\dagger} B_{2} L_{T} \\
& +A_{2}^{\dagger}\left(\left(I-S^{\dagger}\right) R_{A_{1}} V+S^{\dagger} C_{2}\right) T^{\dagger} B_{2} \\
& +A_{2}^{\dagger} Z B_{2}-A_{2}^{\dagger}\left(I-A_{1} A_{1}^{\dagger}+S^{\dagger} S\right) Z\left(B_{1} B_{1}^{\dagger} B_{2}+T\right)+A_{2}^{\dagger} C_{2}+L_{A_{2}} Y,
\end{aligned}
$$

where

$$
\begin{aligned}
V= & -R_{A_{1}} C_{1} B_{1}^{\dagger} B_{2} L_{T}-R_{A_{1}} R_{A_{2}} R_{S} R_{A_{2}} C_{2} T^{\dagger} T \\
& +R_{A_{1}} Q T^{\dagger} T-R_{A_{1}} R_{A_{2}} R_{S} R_{A_{2}} R_{A_{1}} Q T^{\dagger} T
\end{aligned}
$$

and

$$
\begin{aligned}
W= & -R_{S} R_{A_{2}} R_{A_{1}} C_{1}-S S^{\dagger} C_{2} L_{T} B_{2}^{\dagger} B_{1} \\
& +S S^{\dagger} P B_{1}^{\dagger} B_{1}-S S^{\dagger} P B_{1}^{\dagger} B_{2} L_{T} B_{2}^{\dagger} B_{1},
\end{aligned}
$$

where $P, Q, R$ and $Y$ are arbitrary elements of $\mathcal{B}(\mathcal{F}, \mathcal{K}), \mathcal{B}(\mathcal{G}, \mathcal{K}), \mathcal{B}(\mathcal{F}, \mathcal{H})$ and $\mathcal{B}(\mathcal{L}, \mathcal{K})$, respectively.

Proof. $(i) \Rightarrow(i i)$ : Since the system (1.1) is consistent, there exists $X_{2} \in \mathcal{B}(\mathcal{G}, \mathcal{K})$ such that equations

$$
\begin{aligned}
& A_{1} X_{1}-X_{2} B_{1}=C_{1} \\
& A_{2} X_{3}-X_{2} B_{2}=C_{2}
\end{aligned}
$$

are solvable for $X_{1}$ and $X_{3}$, respectively. According to Lemma 2.1 equation

$$
A_{1} X_{1}-X_{2} B_{1}=C_{1}
$$

is solvable for $X_{1}$ if and only if

$$
\left(I-A_{1} A_{1}^{\dagger}\right)\left(C_{1}+X_{2} B_{2}\right)=0,
$$

and equation

$$
A_{2} X_{3}-X_{2} B_{2}=C_{2}
$$


is solvable for $X_{2}$ if and only if

$$
\left(I-A_{2} A_{2}^{\dagger}\right)\left(C_{2}+X_{2} B_{2}\right)=0 .
$$

So, from (2.1) and (2.2) it follows that equations

$$
\begin{aligned}
& \left(I-A_{1} A_{1}^{\dagger}\right) X_{2} B_{1}=-\left(I-A_{1} A_{1}^{\dagger}\right) C_{1}, \\
& \left(I-A_{2} A_{2}^{\dagger}\right) X_{2} B_{2}=-\left(I-A_{2} A_{2}^{\dagger}\right) C_{2}
\end{aligned}
$$

have a common solution. From Lemma 2.1 and Lemma 2.2 system (2.3) is consistent if and only if

$$
\begin{aligned}
& \left(I-A_{1} A_{1}^{\dagger}\right) C_{1}\left(I-B_{1}^{\dagger} B_{1}\right)=0 \\
& \left(I-A_{2} A_{2}^{\dagger}\right) C_{2}\left(I-B_{2}^{\dagger} B_{2}\right)=0 \\
& \left(I-S S^{\dagger}\right) C=0 .
\end{aligned}
$$

(ii) $\Rightarrow($ i): If $(i i)$ holds, then by Lemma 2.2 it follows that system (2.3) is consistent. Let $X_{2} \in \mathcal{B}(G, K)$ be the solution to the system (2.3) and let $X_{1}=$ $A_{1}^{\dagger}\left(X_{2} B_{1}+C_{1}\right)$ and $X_{3}=A_{2}^{\dagger}\left(X_{2} B_{2}+C_{2}\right)$. Then it is easy to see that such $X_{1}, X_{2}$ and $X_{3}$ satisfy (1.1).

(ii) $\Rightarrow($ iii): Suppose that

$$
\begin{aligned}
& \left(I-A_{1} A_{1}^{\dagger}\right) C_{1}\left(I-B_{1}^{\dagger} B_{1}\right)=0, \\
& \left(I-A_{1} A_{1}^{\dagger}\right) C_{1}\left(I-B_{1}^{\dagger} B_{1}\right)=0
\end{aligned}
$$

and

$$
\left(I-S S^{\dagger}\right) C=0
$$

hold. From (2.6) we get

$$
\begin{aligned}
& C\left(I-\left(B_{2} L_{T}\right)^{\dagger}\left(B_{2} L_{T}\right)\right) \\
= & C\left(I-\left(B_{2}\left(I-T^{\dagger} T\right)\right)^{\dagger}\left(B_{2}\left(I-T^{\dagger} T\right)\right)\right) \\
= & \left(I-A_{2} A_{2}^{\dagger}\right) C_{2}\left(I-T^{\dagger} T\right)\left(I-\left(B_{2}\left(I-T^{\dagger} T\right)\right)^{\dagger}\left(B_{2}\left(I-T^{\dagger} T\right)\right)\right) \\
& -\left(I-A_{2} A_{2}^{\dagger}\right)\left(I-A_{1} A_{1}^{\dagger}\right) C_{1} B_{1}^{\dagger} B_{2}\left(I-T^{\dagger} T\right)\left(I-\left(B_{2}\left(I-T^{\dagger} T\right)\right)^{\dagger}\left(B_{2}\left(I-T^{\dagger} T\right)\right)\right) \\
= & \left(I-A_{2} A_{2}^{\dagger}\right) C_{2}\left(I-T^{\dagger} T\right)\left(I-\left(B_{2}\left(I-T^{\dagger} T\right)\right)^{\dagger}\left(B_{2}\left(I-T^{\dagger} T\right)\right)\right) \\
= & \left(I-A_{2} A_{2}^{\dagger}\right) C_{2} B_{2}^{\dagger} B_{2}\left(I-T^{\dagger} T\right)\left(I-\left(B_{2}\left(I-T^{\dagger} T\right)\right)^{\dagger}\left(B_{2}\left(I-T^{\dagger} T\right)\right)\right) \\
= & 0 .
\end{aligned}
$$

$($ iii $) \Rightarrow(i i)$ : Suppose that

$$
\left(I-A_{1} A_{1}^{\dagger}\right) C_{1}\left(I-B_{1}^{\dagger} B_{1}\right)=0,
$$




$$
C\left(I-\left(B_{2}\left(I-T^{\dagger} T\right)\right)^{\dagger}\left(B_{2}\left(I-T^{\dagger} T\right)\right)\right)=0
$$

and

$$
\left(I-S S^{\dagger}\right) C=0
$$

hold. From (2.8) we get

$$
\begin{aligned}
& R_{A_{2}} C_{2}\left(I-T^{\dagger} T\right)\left(I-\left(B_{2}\left(I-T^{\dagger} T\right)\right)^{\dagger}\left(B_{2}\left(I-T^{\dagger} T\right)\right)\right) \\
= & R_{A_{2}} R_{A_{1}} C_{1} B_{1}^{\dagger} B_{2}\left(I-T^{\dagger} T\right) L_{B_{2}\left(I-T^{\dagger} T\right)} \\
= & 0 .
\end{aligned}
$$

Note that

$$
\begin{aligned}
& \left(I-T^{\dagger} T\right) L_{B_{2}} \\
= & \left(I-\left(\left(I-B_{1} B_{1}^{\dagger}\right) B_{2}\right)^{\dagger}\left(I-B_{1} B_{1}^{\dagger}\right) B_{2}\right)\left(I-B_{2}^{\dagger} B_{2}\right) \\
= & I-B_{2}^{\dagger} B_{2} \\
= & L_{B_{2}},
\end{aligned}
$$

so from (2.11) and (2.10) we get

$$
\begin{aligned}
& R_{A_{2}} C_{2} L_{B_{2}} \\
= & R_{A_{2}} C_{2}\left(I-T^{\dagger} T\right) L_{B_{2}} \\
= & R_{A_{2}} C_{2}\left(I-T^{\dagger} T\right)\left(B_{2}\left(I-T^{\dagger} T\right)\right)^{\dagger} B_{2}\left(I-T^{\dagger} T\right) L_{B_{2}} \\
= & R_{A_{2}} C_{2}\left(I-T^{\dagger} T\right)\left(B_{2}\left(I-T^{\dagger} T\right)\right)^{\dagger}\left(I-T^{\dagger} R_{B_{1}}\right) B_{2} L_{B_{2}} \\
= & 0 .
\end{aligned}
$$

Suppose that system (1.1) is consistent.

Since $X_{2} \in \mathcal{B}(G, K)$ is a solution to (1.1) if and only if it satisfies (2.3), its general form, according to Lemma 2.2, is given by

$$
\begin{aligned}
X_{2}= & \left(-R_{A_{1}} C_{1}+S^{\dagger}\left(R_{A_{1}} C_{1}+W\right)\right) B_{1}^{\dagger}\left(I-B_{2} T^{\dagger}\right) \\
& +\left(\left(I-S^{\dagger}\right) R_{A_{1}} V-S^{\dagger} C_{2}\right) T^{\dagger} \\
& +Z-\left(I-A_{1} A_{1}^{\dagger}+S^{\dagger} S\right) Z\left(B_{1} B_{1}^{\dagger}+T T^{\dagger}\right),
\end{aligned}
$$

where $Z$ is an arbitrary element of $\mathcal{B}(\mathcal{G}, \mathcal{K})$, and

$$
\begin{aligned}
V= & -R_{A_{1}} C_{1} B_{1}^{\dagger} B_{2} L_{T}-R_{A_{1}} R_{A_{2}} R_{S} R_{A_{2}} C_{2} T^{\dagger} T \\
& +R_{A_{1}} Q T^{\dagger} T-R_{A_{1}} R_{A_{2}} R_{S} R_{A_{2}} R_{A_{1}} Q T^{\dagger} T
\end{aligned}
$$

and

$$
\begin{aligned}
W= & -R_{S} R_{A_{2}} R_{A_{1}} C_{1}-S S^{\dagger} C_{2} L_{T} B_{2}^{\dagger} B_{1} \\
& +S S^{\dagger} P B_{1}^{\dagger} B_{1}-S S^{\dagger} P B_{1}^{\dagger} B_{2} L_{T} B_{2}^{\dagger} B_{1},
\end{aligned}
$$


where $P$ and $Q$ are arbitrary elements of $\mathcal{B}(\mathcal{F}, \mathcal{K})$ and $\mathcal{B}(\mathcal{G}, \mathcal{K})$.

From the first equation in (1.1) we have

$$
A_{1} X_{1}=X_{2} B_{1}+C_{1},
$$

so, by Lemma 2.1 we get

$$
\begin{aligned}
X_{1} & =A_{1}^{\dagger}\left(X_{2} B_{1}+C_{1}\right)+L_{A_{1}} R \\
& =A_{1}^{\dagger} S^{\dagger}\left(R_{A_{1}} C_{1}+W\right) B_{1}^{\dagger} B_{1}+A_{1}^{\dagger} Z B_{1}-A_{1}^{\dagger} S^{\dagger} S Z B_{1}+A_{1}^{\dagger} C_{1}+L_{A_{1}} R,
\end{aligned}
$$

where $R$ is an arbitrary element of $\mathcal{B}(\mathcal{F}, \mathcal{H})$.

From the second equation in (1.1) we have

$$
A_{2} X_{3}=X_{2} B_{2}+C_{2},
$$

so, by Lemma 2.1 we get

$$
\begin{aligned}
X_{3}= & A_{2}^{\dagger}\left(X_{2} B_{2}+C_{2}\right)+L_{A_{2}} Y \\
= & A_{2}^{\dagger}\left(-R_{A_{1}} C_{1}-S^{\dagger}\left(R_{A_{1}} C_{1}+W\right)\right) B_{1}^{\dagger} B_{2} L_{T} \\
& +A_{2}^{\dagger}\left(\left(I-S^{\dagger}\right) R_{A_{1}} V+S^{\dagger} C_{2}\right) T^{\dagger} B_{2} \\
& +A_{2}^{\dagger} Z B_{2}-A_{2}^{\dagger}\left(I-A_{1} A_{1}^{\dagger}+S^{\dagger} S\right) Z\left(B_{1} B_{1}^{\dagger} B_{2}+T\right)+A_{2}^{\dagger} C_{2}+L_{A_{2}} Y,
\end{aligned}
$$

where $Y$ is an arbitrary element of $\mathcal{B}(\mathcal{L}, \mathcal{K})$.

Theorem 2.2. Let $A_{1} \in \mathcal{B}(\mathcal{H}, \mathcal{K}), B_{1} \in \mathcal{B}(\mathcal{M}, \mathcal{L}), C_{1} \in \mathcal{B}(\mathcal{M}, \mathcal{K}), A_{2} \in \mathcal{B}(\mathcal{K}, \mathcal{N})$, $B_{2} \in \mathcal{B}(\mathcal{L}, \mathcal{G}), C_{2} \in \mathcal{B}(\mathcal{L}, \mathcal{N})$ be such that $A_{1}, A_{2}, B_{1}, B_{2}, S$ and $T$ are all regular. Put

$$
\begin{aligned}
& T=\left(I-B_{1} B_{1}^{\dagger}\right)\left(I-B_{2}^{\dagger} B_{2}\right), \quad S=A_{2} A_{1} A_{1}^{\dagger}, \\
& C=\left(I-\left(A_{2} A_{1}\right)\left(A_{2} A_{1}\right)^{\dagger}\right)\left(C_{2}+A_{2}\left(I-A_{1} A_{1}^{\dagger}\right) C_{1} B_{1}^{\dagger}\right)\left(I-B_{2}^{\dagger} B_{2}\right) .
\end{aligned}
$$

The following statements are equivalent:

(i) The system (1.2) is consistent;

(ii) $R_{A_{1}} C_{1} L_{B_{1}}=0, R_{A_{2}} C_{2} L_{B_{2}}=0, C L_{T}=0$;

(iii) $R_{A_{1}} C_{1} L_{B_{1}}=0,\left(I-R_{A_{2} A_{1}} A_{2}\left(R_{A_{2} A_{1}} A_{2}\right)^{\dagger}\right) C=0, C L_{T}=0$.

In this case, the general solution to the system (1.2) is given by

$$
\begin{aligned}
X_{1}= & A_{1}^{\dagger} S^{\dagger} A_{2} R_{A_{1}} C_{1}+A_{1}^{\dagger} S^{\dagger} W B_{1}^{\dagger} B_{1}+A_{1}^{\dagger}\left(I-S^{\dagger}\right) V B_{1} \\
+ & A_{1}^{\dagger} Z B_{1}-A_{1}^{\dagger} S^{\dagger} S Z B_{1}+A_{1}^{\dagger} C_{1}+R_{A_{1}} R, \\
X_{2}= & \left(-R_{A_{1}} C_{1}+S^{\dagger}\left(A_{2} R_{A_{1}} C_{1}+W\right)\right) B_{1}^{\dagger}\left(I-T^{\dagger}\right) \\
& +\left(\left(I-S^{\dagger} A_{2}\right) R_{A_{1}} V+S^{\dagger} C_{2} L_{B_{2}}\right) T^{\dagger} \\
& +Z-\left(R_{A_{1}}+S^{\dagger} S\right) Z\left(B_{1} B_{1}^{\dagger}+T T^{\dagger}\right),
\end{aligned}
$$




$$
\begin{aligned}
X_{3}= & A_{2}\left(-R_{A_{1}} C_{1}+S^{\dagger}\left(A_{2} R_{A_{1}} C_{1}+W\right)\right) B_{1}^{\dagger}\left(I-T^{\dagger}\right) B_{2}^{\dagger} \\
& +A_{2}\left(\left(I-S^{\dagger} A_{2}\right) R_{A_{1}} V+S^{\dagger} C_{2} L_{B_{2}}\right) T^{\dagger} B_{2}^{\dagger} \\
& +A_{2} Z B_{2}^{\dagger}-A_{2}\left(R_{A_{1}}+S^{\dagger} S\right) Z\left(B_{1} B_{1}^{\dagger}+T T^{\dagger}\right) B_{2}^{\dagger}-C_{2} B_{2}^{\dagger}+Y R_{B_{2}},
\end{aligned}
$$

where

$$
V=-R_{A_{1}} C_{1} B_{1}^{\dagger} L_{B_{2}} L_{T}+R_{A_{1}} Q T^{\dagger} T-R_{A_{1}} A_{2}^{\dagger} R_{S} A_{2} R_{A_{1}} Q T^{\dagger} T
$$

and

$$
W=-R_{S} A_{2} R_{A_{1}} C_{1}+S S^{\dagger} C_{2} L_{B_{2}} B_{1}+S S^{\dagger} P B_{1}^{\dagger} B_{1}-S S^{\dagger} P B_{1}^{\dagger} L_{B_{2}} B_{1}
$$

with $P, Q, Z$ and $Y$ arbitrary elements of $\mathcal{B}(\mathcal{F}, \mathcal{K}), \mathcal{B}(\mathcal{N}, \mathcal{K}), \mathcal{B}(\mathcal{G}, \mathcal{K})$, and $\mathcal{B}(\mathcal{N}, \mathcal{M})$, respectively.

Proof. $(i) \Rightarrow(i i)$ : Since the system (1.1) is consistent, there exists $X_{2} \in \mathcal{B}(\mathcal{G}, \mathcal{K})$ such that equations

$$
\begin{aligned}
& A_{1} X_{1}-X_{2} B_{1}=C_{1} \\
& A_{2} X_{2}-X_{3} B_{2}=C_{2}
\end{aligned}
$$

are solvable for $X_{1}$ and $X_{3}$, respectively. According to Lemma 2.1 equation

$$
A_{1} X_{1}-X_{2} B_{1}=C_{1}
$$

is solvable for $X_{1}$ if and only if

$$
\left(I-A_{1} A_{1}^{\dagger}\right)\left(C_{1}+X_{2} B_{2}\right)=0
$$

and equation

$$
A_{2} X_{2}-X_{3} B_{2}=C_{2}
$$

is solvable for $X_{3}$ if and only if

$$
\left(A_{2} X_{2}-C_{2}\right)\left(I-B_{2}^{\dagger} B_{2}\right)=0 .
$$

So, from (2.13) and (2.15) it follows that equations

$$
\begin{aligned}
& \left(I-A_{1} A_{1}^{\dagger}\right) X_{2} B_{1}=-\left(I-A_{1} A_{1}^{\dagger}\right) C_{1}, \\
& A_{2} X_{2}\left(I-B_{2}^{\dagger} B_{2}\right)=C_{2}\left(I-B_{2}^{\dagger} B_{2}\right)
\end{aligned}
$$

have a common solution. From Lemma 2.1 and Lemma 2.2 system (2.16) is consistent if and only if

$$
\begin{aligned}
& \left(I-A_{1} A_{1}^{\dagger}\right) C_{1}\left(I-B_{1}^{\dagger} B_{1}\right)=0, \\
& \left(I-A_{2} A_{2}^{\dagger}\right) C_{2}\left(I-B_{2}^{\dagger} B_{2}\right)=0 \\
& C^{\prime}\left(I-T^{\dagger} T\right)=0
\end{aligned}
$$


where

$$
C^{\prime}=\left(I-S S^{\dagger}\right)\left(C_{2}+A_{2}\left(I-A_{1} A_{1}^{\dagger}\right) C_{1} B_{1}^{\dagger}\right)\left(I-B_{2}^{\dagger} B_{2}\right) .
$$

Note that condition

$$
C^{\prime}\left(I-T^{\dagger} T\right)=0
$$

is equivalent to

$$
C\left(I-T^{\dagger} T\right)=0
$$

since (2.17) implies

$$
\begin{aligned}
& C\left(I-T^{\dagger} T\right) \\
= & R_{A_{2} A_{1}}\left(C_{2}+A_{2}\left(I-A_{1} A_{1}^{\dagger}\right) C_{1} B_{1}^{\dagger}\right) L_{B_{2}} L_{T} \\
= & R_{A_{2} A_{1}} S S^{\dagger}\left(C_{2}+A_{2}\left(I-A_{1} A_{1}^{\dagger}\right) C_{1} B_{1}^{\dagger}\right) L_{B_{2}} L_{T} \\
= & R_{A_{2} A_{1}} A_{2} A_{1} A_{1}^{\dagger} S^{\dagger}\left(C_{2}+A_{2}\left(I-A_{1} A_{1}^{\dagger}\right) C_{1} B_{1}^{\dagger}\right) L_{B_{2}} L_{T} \\
= & 0,
\end{aligned}
$$

and (2.18) implies

$$
\begin{aligned}
& C^{\prime}\left(I-T^{\dagger} T\right) \\
= & R_{S}\left(C_{2}+A_{2}\left(I-A_{1} A_{1}^{\dagger}\right) C_{1} B_{1}^{\dagger}\right) L_{B_{2}} L_{T} \\
= & R_{S}\left(A_{2} A_{1}\right)\left(A_{2} A_{1}\right)^{\dagger}\left(C_{2}+A_{2}\left(I-A_{1} A_{1}^{\dagger}\right) C_{1} B_{1}^{\dagger}\right) L_{B_{2}} L_{T} \\
= & \left(I-\left(A_{2} A_{1} A_{1}^{\dagger}\right)\left(A_{2} A_{1} A_{1}^{\dagger}\right)^{\dagger}\right)\left(A_{2} A_{1}\right)\left(A_{2} A_{1}\right)^{\dagger}\left(C_{2}+A_{2}\left(I-A_{1} A_{1}^{-}\right) C_{1} B_{1}^{-}\right) L_{B_{2}} L_{T} \\
= & 0 .
\end{aligned}
$$

I follows that

$$
\begin{aligned}
& \left(I-A_{1} A_{1}^{\dagger}\right) C_{1}\left(I-B_{1}^{\dagger} B_{1}\right)=0, \\
& \left(I-A_{2} A_{2}^{\dagger}\right) C_{2}\left(I-B_{2}^{\dagger} B_{2}\right)=0, \\
& C\left(I-T^{\dagger} T\right)=0 .
\end{aligned}
$$

$(i i) \Rightarrow(i)$ : If $(i i)$ holds, then by Lemma 2.2 it follows that system $(2.16)$ is consistent. Let $X_{2} \in \mathcal{B}(\mathcal{G}, \mathcal{K})$ be the solution to the system $(2.16)$ and let $X_{1}=$ $A_{1}^{\dagger}\left(X_{2} B_{1}+C_{1}\right)$ and $X_{3}=\left(A_{2} X_{2}-C_{2}\right) B_{2}^{\dagger}$. Then it is easy to see that such $X_{1}, X_{2}$ and $X_{3}$ satisfy (1.2).

$($ ii $) \Rightarrow($ iii $)$ : Suppose that

$$
\begin{aligned}
& \left(I-A_{1} A_{1}^{\dagger}\right) C_{1}\left(I-B_{1}^{\dagger} B_{1}\right)=0, \\
& \left(I-A_{2} A_{2}^{\dagger}\right) C_{2}\left(I-B_{2}^{\dagger} B_{2}\right)=0
\end{aligned}
$$


and

$$
C\left(I-T^{\dagger} T\right)=0
$$

From (2.20) we obtain

$$
\begin{aligned}
& \left(I-R_{A_{2} A_{1}} A_{2}\left(R_{A_{2} A_{1}} A_{2}\right)^{\dagger}\right) C \\
= & \left(I-R_{A_{2} A_{1}} A_{2}\left(R_{A_{2} A_{1}} A_{2}\right)^{\dagger}\right) R_{A_{2} A_{1}}\left(C_{2}+A_{2}\left(I-A_{1} A_{1}^{\dagger}\right) C_{1} B_{1}^{\dagger}\right) L_{B_{2}} \\
= & \left(I-R_{A_{2} A_{1}} A_{2}\left(R_{A_{2} A_{1}} A_{2}\right)^{\dagger}\right) R_{A_{2} A_{1}} C_{2} L_{B_{2}} \\
& +\left(I-R_{A_{2} A_{1}} A_{2}\left(R_{A_{2} A_{1}} A_{2}\right)^{\dagger}\right) R_{A_{2} A_{1}} A_{2}\left(I-A_{1} A_{1}^{\dagger}\right) C_{1} B_{1}^{\dagger} L_{B_{2}} \\
= & \left(I-R_{A_{2} A_{1}} A_{2}\left(R_{A_{2} A_{1}} A_{2}\right)^{\dagger}\right) R_{A_{2} A_{1}} A_{2} A_{2}^{\dagger} C_{2} L_{B_{2}} \\
= & 0 .
\end{aligned}
$$

$($ ii $) \Rightarrow($ iii $)$ : Suppose that

$$
\begin{gathered}
\left(I-A_{1} A_{1}^{\dagger}\right) C_{1}\left(I-B_{1}^{\dagger} B_{1}\right)=0, \\
\left(I-R_{A_{2} A_{1}} A_{2}\left(R_{A_{2} A_{1}} A_{2}\right)^{\dagger}\right) C=0
\end{gathered}
$$

and

$$
C\left(I-T^{\dagger} T\right)=0
$$

From (2.23) we get

$$
\begin{aligned}
& \left(I-A_{2} A_{2}^{\dagger}\right) C_{2}\left(I-B_{2}^{\dagger} B_{2}\right) \\
= & \left(I-A_{2} A_{2}^{\dagger}\right) C \\
= & \left(I-A_{2} A_{2}^{\dagger}\right) R_{A_{2} A_{1}} A_{2}\left(R_{A_{2} A_{1}} A_{2}\right)^{\dagger} C \\
= & 0 .
\end{aligned}
$$

Suppose that system (1.2) is consistent. Since $X_{2} \in \mathcal{B}(G, K)$ is a solution to (1.2) if and only if it is solution to (2.16), its general form, according to Lemma 2.2, is given by

$$
\begin{aligned}
X_{2}= & \left(-R_{A_{1}} C_{1}+S^{\dagger}\left(A_{2} R_{A_{1}} C_{1}+W\right)\right) B_{1}^{\dagger}\left(I-T^{\dagger}\right) \\
& +\left(\left(I-S^{\dagger} A_{2}\right) R_{A_{1}} V+S^{\dagger} C_{2} L_{B_{2}}\right) T^{\dagger} \\
& +Z-\left(R_{A_{1}}+S^{\dagger} S\right) Z\left(B_{1} B_{1}^{\dagger}+T T^{\dagger}\right),
\end{aligned}
$$

where

$$
V=-R_{A_{1}} C_{1} B_{1}^{\dagger} L_{B_{2}} L_{T}+R_{A_{1}} Q T^{\dagger} T-R_{A_{1}} A_{2}^{\dagger} R_{S} A_{2} R_{A_{1}} Q T^{\dagger} T
$$

and

$$
W=-R_{S} A_{2} R_{A_{1}} C_{1}+S S^{\dagger} C_{2} L_{B_{2}} B_{1}+S S^{\dagger} P B_{1}^{\dagger} B_{1}-S S^{\dagger} P B_{1}^{\dagger} L_{B_{2}} B_{1}
$$


with $P, Q, Z$ arbitrary elements of $\mathcal{B}(\mathcal{F}, \mathcal{M}), \mathcal{B}(\mathcal{G}, \mathcal{K})$ and $\mathcal{B}(\mathcal{G}, \mathcal{K})$, respectively.

From the first equation in (1.2) we have

$$
A_{1} X_{1}=X_{2} B_{1}+C_{1},
$$

so, by Lemma 2.1 we get

$$
\begin{aligned}
X_{1} & =A_{1}^{\dagger}\left(X_{2} B_{1}+C_{1}\right)+L_{A_{1}} R \\
& =A_{1}^{\dagger} S^{\dagger}\left(A_{2} R_{A_{1}} C_{1}+W\right) B_{1}^{\dagger} B_{1}+A_{1}^{\dagger} Z B_{1}-A_{1}^{\dagger} S^{\dagger} S Z B_{1}+A_{1}^{\dagger} C_{1}+L_{A_{1}} R,
\end{aligned}
$$

where $R$ is an arbitrary element of $\mathcal{B}(\mathcal{F}, \mathcal{H})$.

From the second equation in (1.2) we have

$$
X_{3} B_{2}=A_{2} X_{2}-C_{2},
$$

so, by Lemma 2.1 we get

$$
\begin{aligned}
X_{3}= & \left(A_{2} X_{2}-C_{2}\right) B_{2}^{\dagger}+Y R_{B_{2}} \\
= & A_{2}\left(-R_{A_{1}} C_{1}+S^{\dagger}\left(A_{2} R_{A_{1}} C_{1}+W\right)\right) B_{1}^{\dagger}\left(I-T^{\dagger}\right) B_{2}^{\dagger} \\
& +A_{2}\left(\left(I-S^{\dagger} A_{2}\right) R_{A_{1}} V+S^{\dagger} C_{2} L_{B_{2}}\right) T^{\dagger} B_{2}^{\dagger} \\
& +A_{2} Z B_{2}^{\dagger}-A_{2}\left(R_{A_{1}}+S^{\dagger} S\right) Z\left(B_{1} B_{1}^{\dagger}+T T^{\dagger}\right) B_{2}^{\dagger}-C_{2} B_{2}^{\dagger}+Y R_{B_{2}},
\end{aligned}
$$

where $Y$ is an arbitrary element of $\mathcal{B}(\mathcal{N}, \mathcal{M})$.

\section{RE F E R E N C E S}

1. A. Ben-Israel, T. N. E. Greville, Generalized Inverse: Theory and Applications, 2nd Edition, Springer, New York, 2003.

2. A. DAJIC, Common solutions of linear equations in ring, with applications, Electron. J. Linear Algebra, 30 (2015), 66-79.

3. S.G. LEE, Q.P. VU, Simultaneous solutions of matrix equations and simultaneous equivalence of matrices, Linear Algebra Appl., 437 (2012), 2325-2339.

4. Y. H. LiU, Ranks of solutions of the linear matrix equation $A X+Y B=C$. Comput. Math. Appl., 52 (2006), 861-872.

5. Q.W. WANG, Z.H. HE, Solvability conditions and general solution for the mixed Sylvester equations, Automatica, 49 (2013), 2713-2719.

6. Z.H. HE, Q.W. WANG, A pair of mixed generalized Sylvester matrix equations, Journal of Shanghai University (Natural Science), 20 (2014), 138-156.

7. Z.-H. He, Q.-W. WANG, Y. Zhang, A system of quaternary coupled Sylvester-type real quaternion matrix equations, Automatica, 87 (2018), 25-31. 